\title{
ON BROWNIAN EXCURSIONS IN LIPSCHITZ DOMAINS. PART I. LOCAL PATH PROPERTIES
}

\author{
KRZYSZTOF BURDZY AND RUTH J. WILLIAMS
}

\begin{abstract}
A necessary and sufficient condition is given for a Brownian excursion law in a Lipschitz domain to share the local path properties with an excursion law in a halfspace. This condition is satisfied for all boundary points of every $C^{1, \alpha}$-domain, $\alpha>0$. There exists a $C^{1}$-domain such that the condition is satisfied almost nowhere on the boundary. A probabilistic interpretation and applications to minimal thinness and boundary behavior of Green functions are given.
\end{abstract}

0. Introduction. We will show that the local path properties of Brownian excursions in $C^{1, \alpha}$-domains, $\alpha>0$, are the same as the local path properties of Brownian excursions in a half-space (Theorem 3.1). This need not be the case if the domain is of class $C^{1}$ (Proposition 3.1). The method of proof is based on the exit system theory of Maisonneuve [12]. We will study Brownian excursion laws, i.e., one of the ingredients of an exit system. We will give a necessary and sufficient condition for an excursion law in a Lipschitz domain to have the same local path properties as an excursion law in a half-space (Theorems 2.1 and 2.2). This last result can be applied to obtain new criteria for minimal thinness (Theorem 4.1) and existence of a nondegenerate normal derivative for the Green function (Theorem 4.2).

Local path properties of 1-dimensional Brownian excursions have been known for some time. They are the same as the local path properties of the 3-dimensional Bessel process (see $\S 2.10$ of Itô and McKean [11] or $\S I I .67$ of Williams [21]). Dvoretsky and Erdös [8] and Shiga and Watanabe [18] have given Kolmogorov-type tests for Bessel processes. Local path properties of excursions of multidimensional Brownian motion have been studied by Shimura [19] (excursions of 2-dimensional Brownian motion from a line) and Burdzy [1] ( $n$-dimensional case, $n \geq 2)$.

Our theorem about minimal thinness generalizes results of Essen and Jackson [9] and Burdzy $[\mathbf{3}, \mathbf{4}]$. A new criterion for the existence of the nondegenerate normal derivative for the Green function extends some results of Widman [20], Rippon [17] and Burdzy [4].

The present article mainly uses ideas from excursion theory (see Maisonneuve [12], Williams [21] and Burdzy [1]) and potential theory (see Doob [7]).

Received by the editors July 26,1985 .

1980 Mathematics Subject Classification (1985 Revision). Primary 60G17, 60J50, 60J65; Secondary 31B25, $60 \mathrm{~J} 45$.

Key words and phrases. Brownian motion, excursions, path properties, minimal thinness, Green functions, Lipschitz domains.

Research supported in part by NSF Grants DMS-8419377 and DMS-8319562. 
1. Notation, definitions and review of relevant results. We will work with the $n$-dimensional Euclidean space $\mathbf{R}^{n}, n \geq 2$ and will use different Cartesian coordinate systems $\mathrm{CS}_{1}, \mathrm{CS}_{2}$, etc. The following notation will be used: $x=\left(x_{1}, \ldots, x_{n}\right), \mathbf{R}_{k}^{n-1}=\left\{x \in \mathbf{R}^{n}: x_{1}=0\right.$ in $\left.\mathrm{CS}_{k}\right\}, D_{*}^{k}=\left\{x \in \mathbf{R}^{n}: x_{1}>0\right.$ in $\mathrm{CS}_{k}$ \}. If the coordinate system is fixed and no confusion may arise we will use symbols $\mathbf{R}^{n-1}$ and $D_{*}$. We will let $\tilde{x}=\operatorname{proj}_{\mathbf{R}_{k}^{n-1}} x$. We will make sure that it is clear which coordinate system is meant when the notation $\left(x_{1}, \ldots, x_{n}\right)$ or $\tilde{x}$ is used. For a set $A \subset \mathbf{R}^{n}$, we will use $A^{c}$ to denote $\mathbf{R}^{n} \backslash A$.

We will say that a function $h: \mathbf{R}^{n-1} \rightarrow \mathbf{R}$ represents a domain $D$ near $x \in \partial D$ in $\mathrm{CS}_{k}$ if there exists a neighborhood $U$ of $x$ such that

$$
\left\{x \in \mathbf{R}^{n}: x_{1}>h(\tilde{x}) \text { in } \mathrm{CS}_{k}\right\} \cap U=D \cap U .
$$

A domain will be called Lipschitz if it is represented by a Lipschitz function near every boundary point $x$ (the function may depend on $x$ ). Similarly a domain will be called $C^{1}\left(C^{1, \alpha}\right)$ if it is represented near every boundary point by a function which has continuous ( $\alpha$-Hölder continuous) first order partial derivatives. Dahlberg [6] proved that the surface area measure and harmonic measure are mutually absolutely continuous in Lipschitz domains. The expression "almost all" will refer to either of these measures. The surface area measure on hyperplanes will be denoted $d x$. We will use the abbreviations $a^{+}=\max (0, a)$ and $a^{-}=-\min (0, a)$.

We will use the canonical probability space $\left(\Omega, \mathbf{F},\left\{\mathbf{F}_{t}\right\}, X\right)$. Here $\Omega$ is the set of all paths (functions) $\omega:[0, \infty) \rightarrow \mathbf{R}^{n} \cup\{\delta\}$ which are $\mathbf{R}^{n}$-valued and continuous on $[0, R)$, and satisfy $\omega(t)=\delta$ for all $t \geq R$. The lifetime $R=R(\omega)$ of a path is the time of the jump to the isolated trap $\delta$ in $\mathbf{R}^{n} \cup\{\delta\}$ (possibly $R=\infty$ ). The process $X$ is defined by $X_{t}(\omega)=\omega(t)$ and associated raw $\sigma$-fields are defined by

$$
\begin{aligned}
& \mathbf{F}_{t}^{0}=\bigcap_{u>t} \sigma\{X(s), 0 \leq s \leq u\}, \\
& \mathbf{F}^{0}=\sigma\{X(s), 0 \leq s<\infty\}
\end{aligned}
$$

$\mathbf{F}$ is the universal completion of $\mathbf{F}^{0}$ and $\mathbf{F}_{t}$ is the universal completion of $\mathbf{F}_{t}^{0}$ in $\mathbf{F}$. $\theta$ will denote the usual shift operator.

A random variable $T$ is called a stopping time if $\{T \leq t\} \in \mathbf{F}_{t}$ for all $t \geq 0$. The pre- $T \sigma$-field $\mathbf{F}_{T}$ is defined by

$$
\mathbf{F}_{T}=\left\{A \in \Omega: A \cap\{T \leq t\} \in \mathbf{F}_{t} \text { for all } t \geq 0\right\} .
$$

The hitting time $T_{A}$ of a set $A$ will be defined as $\inf \left\{t>0: X_{t} \in A\right\}$ where $\inf \phi=$ $\infty$.

The distribution of standard Brownian motion starting from $x$ will be denoted by $P^{x}$. The distribution of Brownian motion starting from $x$ and killed on the boundary of a domain $D$ will be denoted by $P_{D}^{x}$.

DEFINITION 1.1. A $\sigma$-finite measure $H^{x}, x \in \partial D$ on $(\Omega, \mathbf{F})$ is called an excursion law in $D$ if $H^{x}(X(0) \neq x)=0$ and $H^{x}$ is strong Markov for the transition probabilities of Brownian motion in $D$ in the following sense. For all $H^{x}$-a.s. strictly positive stopping times $T, \mathbf{F}_{T}$-measurable nonnegative $a$, and $\mathbf{F}$-measurable nonnegative $b$, we have:

$$
H^{x}\left(a \cdot b\left(\theta_{T}\right)\right)=H^{x}\left(a \cdot P_{D}^{X_{T}}(b)\right) .
$$


There is no natural normalization of infinite measures so "unique" excursion law will mean "unique up to a multiplicative constant" excursion law. The expression $H_{1}^{x}=H_{2}^{x}$ will be understood in the same spirit.

A set $A \subset \mathbf{R}^{n}$ will be called regular for an excursion law $H^{x}$ if $H^{x}\left(T_{A}>0\right)=0$. We will use the probabilistic expression $H^{x}$-a.s. instead of $H^{x}$-a.e.

DEFINITION 1.2. An excursion law $H^{x}$ in a domain $D$ will be called standard if $0<H^{x}\left(T_{B}<\infty\right)<\infty$ for every nonempty open ball $B \subset \bar{B} \subset D$.

THEOREM 1.1. Suppose that $D$ is Lipschitz and $x \in \partial D$. Then there exists a unique standard excursion law $H^{x}$ in $D$.

Proof. See Theorem 4.1 and Corollary 4.1 of Burdzy [1].

The standard excursion law in $D_{*}^{k}$ starting from $x \in \mathbf{R}_{k}^{n-1}$ will be usually denoted by $H_{*}^{x}$.

Consider two domains $D_{1}$ and $D_{2}$ such that $x \in \partial D_{1} \cap \partial D_{2}$. Suppose $H^{x}$ is an excursion law in $D_{1}$. Kill $H^{x}$-excursions at $T_{D_{2}^{c}}$ on the set where $R \geq T_{D_{2}^{c}}$. On the set where $R<T_{D_{2}^{c}}$ continue the $H^{x}$-excursions as an independent Brownian motion in $D_{2}$ starting from $X(R-)$.

DEFINITION 1.3. The distribution of paths obtained by killing and continuing as described above will be called $D_{1}-D_{2}$ KAC- $H^{x}$.

By the 0-0 law for standard excursion laws (Lemma 4.1 of Burdzy [1]) we have either $T_{D_{2}^{c}}=0 H^{x}$-a.s. or $T_{D_{2}^{c}}>0 H^{x}$-a.s. provided $H^{x}$ is standard. Thus if $H^{x}$ is standard then either $D_{1}-D_{2} \mathrm{KAC}-H^{x}$ is concentrated on $\{\delta\}$ or it is an excursion law in $D_{2}$. The excursion law $D_{1}-D_{2} \mathrm{KAC}-H^{x}$ need not be standard even if $H^{x}$ is standard (see Remark 5.1(ii) of Burdzy [2]).

DEFINITION 1.4. Excursion laws $H_{1}^{x}$ and $H_{2}^{x}$ (not necessarily in the same domain) are said to share the local properties if they are mutually absolutely continuous on $[0, T)$ for some stopping time $T$ which is strictly positive $H_{1}^{x}$-a.s. and $H_{2}^{x}$-a.s.

If excursion laws $H_{1}^{x}$ and $H_{2}^{x}$ are such that $H_{2}^{x}=D_{1}-D_{2} \mathrm{KAC}-H_{1}^{x}$ and $H_{1}^{x}$ is standard then they share the local properties (use $T=\min \left(R, T_{D_{1}^{c}}, T_{D_{2}^{c}}\right)$ ). On the other hand, suppose $H_{1}^{x}$ and $H_{2}^{x}$ are standard excursion laws in domains $D_{1}$ and $D_{2}$, respectively, and they share the local properties. Then for $D_{3} \equiv D_{1} \cap D_{2} \neq$ $\varnothing, H_{3}^{x} \equiv D_{1}-D_{3} \mathrm{KAC}-H_{1}^{x}$ is a standard excursion law in $D_{3}$, and $D_{3}-D_{2} \mathrm{KAC}$ $H_{3}^{x}=H_{2}^{x}$. It follows from this that $D_{1}-D_{2} \mathrm{KAC}-H_{1}^{x}=H_{2}^{x}$.

DEFINITION 1.5. An excursion law $H^{x}$ is called locally flat if $H^{x}=D_{*}-D \mathrm{KAC}$ $H_{*}^{x}$, where $D$ is a domain and $H_{*}^{x}$ is the standard excursion law in a half-space $D_{*}$.

We do not require in the above definition that $H^{x}$ is standard.

Fix a Lipschitz region $D$ and define excursions $\left\{e_{t}(s), s \geq 0\right\}$ of Brownian motion in $D$ as follows. For each $t \geq 0$ such that $X(t) \in D^{c}$, let

$$
e_{t}(s)= \begin{cases}X(t+s) & \text { if } T_{D^{c}} \circ \theta_{t}>s \\ \delta & \text { otherwise. }\end{cases}
$$

The above definition allows for constant excursions $e_{t}(s) \equiv \delta$. The following "exit system" formula is a special case of results of Maisonneuve [12]. Here $L_{t}$ is a local time on $\partial D$ of Brownian motion, i.e., a continuous additive functional with 1-potential $E^{*}\left(e^{-T_{\partial D}}\right)$, and $\tau_{t}=\inf \left\{s \geq 0: L_{s}>t\right\}$. 
THEOREM 1.2. There exists a universally measurable family $\left\{H^{x}\right\}, x \in \mathbf{R}^{n}$ of $\sigma$-finite measures such that

$$
\begin{aligned}
E^{\cdot}\left(\sum_{\substack{0<u<\infty \\
x_{u} \in \partial D}} Z_{u}^{\cdot}\left(f \circ e_{u}\right)\right) & =E^{\cdot}\left(\int_{0}^{\infty} Z_{s} H^{X_{s}}(f) d L_{s}\right) \\
& =E^{\cdot}\left(\int_{0}^{L_{\infty}} Z_{\tau(u)} H^{X_{\tau(u)}}(f) d u\right)
\end{aligned}
$$

for all $\left\{\mathbf{F}_{t}\right\}$-predictable processes $Z$ and universally measurable functions $f$ on $\Omega$, such that $f$ is zero on constant excursions equal to $\delta$. For each $z \in \partial D$, either $H^{z}$ is a standard excursion law in $D$ or $H^{z} \equiv 0$. $D$.

Every pair $(d L, H)$ satisfying the above theorem will be called an exit system in

REMARK 1.1. Suppose that some property holds $H^{x}$-a.s. for $\mu$-almost all $x$, where $\mu$ is the measure associated with $L$ (see Revuz $[15,16]$ ). Then the exit system formula shows that for each $z \in \mathbf{R}^{n}, P^{z}$-a.s. the excursions of Brownian motion in $D$ have the same property.

2. Criteria for local flatness of excursion laws. Suppose that a Lipschitz function $h$ represents the boundary of a domain $D$ near the point $0 \in \partial D$. Let $D_{*}=\left\{x \in \mathbf{R}^{n}: x_{1}>0\right\}$ and $H_{*}^{0}$ be the standard excursion law in $D_{*}$.

THEOREM 2.1. The standard excursion law $H^{0}$ in $D$ shares the local properties with $H_{*}^{0}$ if and only if

$$
\int_{\mathbf{R}^{n-1} \cap\{|x| \leq 1\}} \frac{|h(x)|}{|x|^{n}} d x<\infty .
$$

PROOF. (i) If

$$
\int_{\mathbf{R}^{n-1} \cap\{|x| \leq 1\}} \frac{h^{+}(x)}{|x|^{n}} d x=\infty
$$

then $H_{*}^{0}$-excursions hit $\partial D$ immediately. This follows from Theorem 3.2 and Remark 3.2 of Burdzy [3]. Therefore $H^{0}$ and $H_{*}^{0}$ do not share the local properties in this case.

(ii) Assume now that (2.1) holds. It follows that

$$
\int_{\mathbf{R}^{n-1} \cap\{|x| \leq 1\}} \frac{h^{-}(x)}{|x|^{n}} d x<\infty .
$$

Define $D_{1}=D_{*} \cup D$. Let $H_{1}^{0}$ be the standard excursion law in $D_{1}$. By Theorem 4.5 of Burdzy [1] and Corollary 4.2 of Burdzy [4], (2.3) implies that the excursion laws $H_{1}^{0}$ and $H_{*}^{0}$ share the local properties. Let $K=D_{*} \backslash D=D_{1} \backslash D$. In the present case (2.2) is not satisfied, so Theorem 3.2 and Remark 3.2 of Burdzy [3] imply that $H_{*}^{0}\left(T_{K}=0\right)=0$ and therefore $H_{1}^{0}\left(T_{K}=0\right)=0$. It follows that $D_{1}$ $D$ KAC- $H_{1}^{0}$ is a standard excursion law in $D$ which shares the local properties with $H_{1}^{0}$ and therefore with $H_{*}^{0}$. By the uniqueness of the standard excursion law we have $H^{0}=D_{1}-D \mathrm{KAC}-H_{1}^{0}$ and hence the "if" part of the theorem holds. 
(iii) Suppose that (2.1) does not hold. Then either (2.2) holds and by (i), $H^{0}$ and $H_{*}^{0}$ do not share the local properties, or both (2.2) and (2.3) fail to hold. Thus, to complete the proof of the theorem, we assume (2.2) and (2.3) do not hold. Let $D_{2}=D \cap D_{*}$ and $H_{2}^{0}$ be the standard excursion law in $D_{2}$. We will prove that $H_{3}^{0} \stackrel{\text { df }}{=} D_{2}-D$ KAC- $H_{2}^{0}$ is not standard and show that this implies that $H^{0}$ and $H_{*}^{0}$ do not share the local properties. This part of the proof is the longest and most complicated.

Suppose that $h$ is Lipschitz with constant $K$, i.e., $|h(z)-h(y)| \leq K|z-y|$. For $x \in D_{*}$, let $\hat{x}=\left(-x_{1} / 4, x_{2}, \ldots, x_{n}\right), r_{1}=\min \left(x_{1} / 16, x_{1} / 8 K\right)$ and $r_{2}=2 r_{1}$. Let

$$
B(y, r)=\left\{z \in \mathbf{R}^{n}:|z-y|=r\right\} .
$$

Define $C_{1}(x)=B\left(x, r_{1}\right), C_{2}(x)=B\left(\hat{x}, r_{1}\right), C_{3}(x)=B\left(x, r_{2}\right)$ and $C_{4}(x)=B\left(\hat{x}, r_{2}\right)$. Let $M(x)$ be the interior of the smallest rectangle with sides parallel to the axes which contains $C_{3}(x)$ and $C_{4}(x)$. Since $h$ represents $\partial D$ near 0 it is easy to see that we may choose $\varepsilon>0$ so small that $M(x) \subset D$ for all $x \in Q$, where $Q=$ $\left\{x \in D: x_{1}=-h(\tilde{x})>0\right.$ and $\left.|x| \leq \varepsilon\right\}$. For a fixed $t>0$ define $T_{1}(t)=T_{1}=$ $t+T_{Q} \circ \theta_{t}$, and for $k \geq 1$, define $Q_{k}$ and $T_{k+1}$ inductively by

$$
\begin{aligned}
& Q_{k}(t)=Q_{k}=Q \backslash \bigcup_{i \leq k} M\left(X\left(T_{i}\right)\right) \quad \text { if } T_{k}<\infty, \\
& T_{k+1}(t)=T_{k+1}= \begin{cases}T_{k}+T_{Q_{k}} \circ \theta_{T_{k}} & \text { if } T_{k}<\infty, \\
\infty & \text { otherwise. }\end{cases}
\end{aligned}
$$

Let $B \subset D_{2}$ be a nonempty open ball. Then $H_{2}^{0}\left(T_{B}<\infty\right)>0$. Define events

$$
A_{k}(t)=A_{k}=\left\{T_{k}<\infty, T_{B} \circ \theta_{T_{k}}<\infty\right\} .
$$

Fix an arbitrary integer $m>0$ and an arbitrary real number $\varepsilon_{0}>0$. The excursion law $H_{2}^{0}$ shares the local properties with $H_{*}^{0}$. Since (2.3) holds with $h^{+}$in place of $h^{-}$, this follows from Theorem 4.5 of Burdzy [1] and Corollary 4.2 of Burdzy [4]. We have assumed that

$$
\int_{\mathbf{R}^{n-1} \cap\{|x| \leq 1\}} \frac{h^{-}(x)}{|x|^{n}} d x=\infty .
$$

By Lemma 3.1 of Burdzy [3] and Example 1.XII.12(b) of Doob [7] there is a truncated cone

$$
V=\left\{x \in \mathbf{R}^{n}:|x|<\varepsilon, x_{1}>a|\tilde{x}|\right\} \subset D_{*} \backslash Q
$$

for some $a>0, \varepsilon>0$, such that $H_{*}^{0}\left(T_{V} \neq 0\right)=0$. Thus, $H_{*}^{0}$-a.s., excursions immediately hit the set $V$ above $Q$ and by Theorem 3.2 and Remark 3.2 of Burdzy [3] they immediately hit a set below $Q$, so it follows that $H_{*}^{0}\left(T_{Q} \neq 0\right)=0$ and therefore $H_{2}^{0}\left(T_{Q} \neq 0\right)=0$. Hence, for each fixed $k, 1_{A_{k}(t)} \rightarrow 1_{\left\{T_{B}<\infty\right\}} H_{2}^{0}$-a.s. as $t \rightarrow 0$. Combining this with $1_{A_{k}(t)} \leq 1_{\left\{T_{B}<\infty\right\}}$, it follows that $H_{2}^{0}\left(A_{k}(t)\right) \rightarrow$ $H_{2}^{0}\left(T_{B}<\infty\right)$ as $t \rightarrow 0$. Choose $t>0$ so small that

$$
H_{2}^{0}\left(A_{k}\right) \geq\left(1-\varepsilon_{0}\right) H_{2}^{0}\left(T_{B}<\infty\right)
$$

for all $k \leq m$. Define events $\tilde{A}_{k}$ for $k \geq 1$ by

$$
\begin{array}{r}
\tilde{A}_{k}=\left\{T_{k}<\infty \text { and } S_{k} \stackrel{\text { df }}{=} T_{k}+T\left(C_{2}\left(X\left(T_{k}\right)\right)\right) \circ \theta_{T_{k}}<T_{k}+T\left(\partial M\left(X\left(T_{k}\right)\right)\right) \circ \theta_{T_{k}}\right. \\
\text { and } \left.T_{B} \circ \theta_{S_{k}}<T\left(\partial\left(M\left(X\left(T_{k}\right)\right) \cup D_{2}\right)\right) \circ \theta_{S_{k}}\right\} .
\end{array}
$$


For $z \in Q$, let

$$
p=p(z)=P_{M(z)}^{z}\left(T_{C_{2}(z)}<\infty, T_{C_{1}(z)} \circ \theta\left(T_{C_{2}(z)}\right)<\infty\right) .
$$

Now $p>0$ and by Brownian scaling, $p$ does not depend on $z$. The Harnack inequality applied to the harmonic function $P_{D_{2}}\left(T_{B}<\infty\right)$ in the interior of $C_{3}(x)$ shows that for all $y \in C_{1}(x)$

$$
P_{D_{2}}^{y}\left(T_{B}<\infty\right) \geq c P_{D_{2}}^{x}\left(T_{B}<\infty\right)
$$

where $c>0$ does not depend on $x$ or $y$. For $x \in Q$, define

$$
\hat{T}(x)= \begin{cases}T_{C_{2}(x)}+T_{C_{1}(x)} \circ \theta\left(T_{C_{2}(x)}\right) & \text { if } T_{C_{2}(x)}<\infty \\ \infty & \text { otherwise }\end{cases}
$$

The strong Markov property applied at $T_{k}$ and $T_{k}+\hat{T}\left(X\left(T_{k}\right)\right) \circ \theta_{T_{k}}$ implies that

$$
\begin{aligned}
H_{3}^{0}\left(\tilde{A}_{k}\right) & \geq \int_{Q} \int_{C_{1}(x)} P_{D_{2}}^{y}\left(T_{B}<\infty\right) P_{M(x)}^{x}(X(\hat{T}(x)) \in d y) H_{3}^{0}\left(X\left(T_{k}\right) \in d x\right) \\
& \geq \int_{Q} \int_{C_{1}(x)} P_{D_{2}}^{y}\left(T_{B}<\infty\right) P_{M(x)}^{x}(X(\hat{T}(x)) \in d y) H_{2}^{0}\left(X\left(T_{k}\right) \in d x\right) \\
& \geq \int_{Q} \int_{C_{1}(x)} c P_{D_{2}}^{x}\left(T_{B}<\infty\right) P_{M(x)}^{x}(X(\hat{T}(x)) \in d y) H_{2}^{0}\left(X\left(T_{k}\right) \in d x\right) \\
& =\int_{Q} c P_{D_{2}}^{x}\left(T_{B}<\infty\right) p H_{2}^{0}\left(X\left(T_{k}\right) \in d x\right) \\
& =c p H_{2}^{0}\left(A_{k}\right) \geq c p\left(1-\varepsilon_{0}\right) H_{2}^{0}\left(T_{B}<\infty\right) .
\end{aligned}
$$

The last inequality holds for a fixed $t=t\left(\varepsilon_{0}, m\right)$ and all $k \leq m$. For $t$ fixed, the events $\tilde{A}_{k}, k=1, \ldots, m$, are disjoint and therefore

$$
H_{3}^{0}\left(T_{B}<\infty\right) \geq \sum_{k=1}^{m} H_{3}^{0}\left(\tilde{A}_{k}\right) \geq m c p\left(1-\varepsilon_{0}\right) H_{2}^{0}\left(T_{B}<\infty\right) .
$$

The above formula holds for arbitrary $m$ and $\varepsilon_{0}$ so $H_{3}^{0}\left(T_{B}<\infty\right)=\infty$, i.e., $H_{3}^{0}$ is not standard.

Suppose now that $H_{*}^{0}$ and $H^{0}$ share the local properties. Then

$$
H^{0}\left(T_{\mathbf{R}^{n-1}}=0\right)=0
$$

and $H_{4}^{0} \stackrel{\mathrm{df}}{=} D-D_{2} \mathrm{KAC}-H^{0}$ is a standard excursion law in $D_{2}$. One has therefore $H^{0}=D_{2}-D$ KAC- $H_{4}^{0}$. By the uniqueness of the standard excursion laws we have $H_{4}^{0}=H_{2}^{0}$ and $H^{0}=D_{2}-D \mathrm{KAC}-H_{2}^{0}=H_{3}^{0}$. It has been proved that $H_{3}^{0}$ is not standard which contradicts the assumption that $H^{0}$ is standard. This contradiction proves that $H^{0}$ and $H_{*}^{0}$ do not share the local properties.

REMARKS 2.1. (i) In part (iii) above it has been proved implicitly that $H^{0}\left(T_{\mathbf{R}^{n-1}} \neq 0\right)=0$. This property is in fact equivalent to $R=L_{\mathbf{R}^{n-1}}$ for the Brownian motion in $D$ starting at a point $x \in D$ and conditioned to converge to $0 \in \partial D$. Here $L_{\mathbf{R}^{n-1}}$ denotes the last exit time from $\mathbf{R}^{n-1}$. To prove that $R=L_{\mathbf{R}^{n-1}}$ for the conditioned Brownian motion one may use a Borel-Cantelli Lemma for slightly dependent events (see for example Proposition 3.1 of Port and Stone [14]). This requires finding a suitable sequence of events $\left\{B_{k}\right\}$ replacing $\left\{\tilde{A}_{k}\right\}$ in the above proof and finding probabilities of $B_{k}$ 's and their intersections. 
This seems like a difficult task in view of the fact that transition probabilities and hitting probabilities are not given explictly for conditioned Brownian motion in an arbitrary domain. The approach taken in the above proof is easier because: (i) it requires a sequence of disjoint events $\left\{\tilde{A}_{k}\right\}$ (instead of "almost independent" events $\left\{B_{k}\right\}$ ), and (ii) transition and hitting probabilities for an excursion law correspond to Brownian motion (not conditioned Brownian motion). Thus it pays sometimes to use $\sigma$-finite measures instead of probability measures.

(ii) The method of proof applied in part (iii) above was used earlier in Theorem 7.1 of Burdzy [5] to obtain a result about angular derivatives.

Suppose that a Lipschitz function $h$ represents $D$ near a point $x_{0} \in \partial D$ in a coordinate system $\mathrm{CS}_{1}$. By first performing a translation of coordinates, we may and do assume that $x_{0}=0$ in $\mathrm{CS}_{1}$. Let $H^{x_{0}}$ be the standard excursion law from $x_{0}$ in $D$.

THEOREM 2.2. The excursion law $H^{x_{0}}$ is locally flat if and only if the function $h$ has a total derivative at $0 \in \mathbf{R}_{1}^{n-1}$ and

$$
\int_{\mathbf{R}_{1}^{n-1} \cap\{|x| \leq 1\}} \frac{|h(x)-\nabla h(0) \cdot x|}{|x|^{n}} d x<\infty .
$$

Suppose that $x_{0}=0$ in a coordinate system $\mathrm{CS}_{2}$ and $D_{*}^{2}=\left\{x: x_{1}>v \cdot \tilde{x}\right.$ in $\left.\mathrm{CS}_{1}\right\}$ for some vector $v \in \mathbf{R}_{1}^{n-1}$. If $h$ is a $C^{1}$ function, then for $v=\nabla h(0)$, there is a $C^{1}$ function $g$ representing $D$ in $\mathrm{CS}_{2}$ near $x_{0}$, and one can apply Theorem 2.1 to $g$ to deduce Theorem 2.2. However, if $h$ is merely Lipschitz (or differentiable but not continuously so), then $D$ need not be representable in $\mathrm{CS}_{2}$ near 0 , by any function, Lipschitz or not. For this reason, we prove the following lemma before Theorem 2.2 .

LEMMA 2.1. (i) If

$$
\int_{\mathbf{R}_{1}^{n-1} \cap\{|x| \leq 1\}} \frac{(h(x)-v \cdot x)^{+}}{|x|^{n}} d x<\infty
$$

then there exists a nonnegative Lipschitz function $g_{1}$ which represents a domain $D_{1}$ near $x_{0}$ in $\mathrm{CS}_{2}$ and such that for some neighborhood $U$ of $x_{0}$ we have

$$
D_{1} \cap U \subset D \cap U \text { and } \int_{\mathbf{R}_{2}^{n-1} \cap\{|x| \leq 1\}} \frac{g_{1}(x)}{|x|^{n}} d x<\infty \text {. }
$$

(ii) If

$$
\int_{\mathbf{R}_{1}^{n-1} \cap\{|x| \leq 1\}} \frac{(h(x)-v \cdot x)^{+}}{|x|^{n}} d x=\infty
$$

then there exists a nonnegative Lipschitz function $g_{2}$ which represents a domain $D_{2}$ near $x_{0}$ in $\mathrm{CS}_{2}$ and such that for some neighborhood $U$ of $x_{0}$ we have

$$
D \cap U \cap D_{*}^{2} \subset D_{2} \cap U \cap D_{*}^{2}
$$

and

$$
\int_{\mathbf{R}_{2}^{n-1} \cap\{|x| \leq 1\}} \frac{g_{2}(x)}{|x|^{n}} d x=\infty
$$


(iii) If

$$
\int_{\mathbf{R}_{1}^{n-1} \cap\{|x| \leq 1\}} \frac{(h(x)-v \cdot x)^{-}}{|x|^{n}} d x<\infty
$$

then there exists a nonpositive Lipschitz function $g_{3}$ which represents a domain $D_{3}$ near $x_{0}$ in $\mathrm{CS}_{2}$ and such that for some neighborhood $U$ of $x_{0}$ we have

$$
D \cap U \subset D_{3} \cap U \text { and } \int_{\mathbf{R}_{2}^{n-1} \cap\{|x| \leq 1\}} \frac{\left|g_{3}(x)\right|}{|x|^{n}} d x<\infty .
$$

PROOF. The proofs of the three assertions are totally elementary and very similar to one another so we will sketch only the proof of the existence of $g_{1}$.

Assume that (2.5) holds. This implies that

$$
\lim _{|x| \rightarrow 0}(h(x)-v \cdot x)^{+}|x|^{-1}=0 .
$$

To see this suppose that

$$
\limsup _{|x| \rightarrow 0}(h(x)-v \cdot x)^{+}|x|^{-1}=a>0 .
$$

This implies that there exists a sequence $x_{m} \in \mathbf{R}_{1}^{n-1}$ and $m \geq 1$ such that $\left(h\left(x_{m}\right)-v \cdot x_{m}\right)^{+}\left|x_{m}\right|^{-1}>a / 2$ and $2^{-k_{m}-1} \leq\left|x_{m}\right| \leq 2^{-k_{m}}$, where $\left\{k_{m}\right\}$ is an increasing sequence of integers such that $k_{m+1}-k_{m} \geq 3$. The function $(h(x)-v \cdot x)^{+}$ is Lipschitz with a constant $K_{1}>1$ so $(h(x)-v \cdot x)^{+}|x|^{-1}>a / 16$ for all $x$ in the set $W_{m}=\left\{z \in \mathbf{R}_{1}^{n-1}:\left|z-x_{m}\right|<r\right\}$ where $r=2^{-k_{m}} \min \left(a / 8 K_{1}, 1 / 4\right)$. The volume of $W_{m}$ is equal to $c \cdot r^{n-1}$ so we have

$$
\begin{aligned}
\int_{W_{m}} \frac{(h(x)-v \cdot x)^{+}}{|x|^{n}} d x & \geq \frac{a}{16} \cdot\left(2^{-k_{m}+1}\right)^{-n+1} \cdot c r^{n-1} \\
= & \frac{a}{16} \cdot\left(2^{-k_{m}+1}\right)^{-n+1} \cdot c\left(2^{-k_{m}} \min \left(\frac{a}{8 K_{1}}, \frac{1}{4}\right)\right)^{n-1}=c_{1}>0
\end{aligned}
$$

where $c_{1}$ does not depend on $m$. The sets $W_{m}$ are disjoint so

$$
\begin{aligned}
\int_{\mathbf{R}_{1}^{n-1} \cap\{|x| \leq 1\}} \frac{(h(x)-v \cdot x)^{+}}{|x|^{n}} d x & \geq \sum_{m=1}^{\infty} \int_{W_{m}} \frac{(h(x)-v \cdot x)^{+}}{|x|^{n}} d x \\
& \geq \sum_{m=1}^{\infty} c_{1}=\infty .
\end{aligned}
$$

This contradicts (2.5) and we conclude that (2.8) holds.

Choose $j$ so large that

$$
(h(x)-v \cdot x)^{+}|x|^{-1}<2^{-3}(n-1)^{-1 / 2}
$$

for all $x$ in the set

$$
Q=\left\{x \in \mathbf{R}_{1}^{n-1}:\left|x_{k}\right| \leq 2^{-j}, k=2, \ldots, n\right\} .
$$

For $i>j$ define inductively sequences of sets $Q_{m_{i}+1}, \ldots, Q_{m_{i+1}}$ where $m_{j+1}=0$. For each $i>j$ let $Q_{m_{i}+1}, \ldots, Q_{m_{i+1}}$ be the sequence of all sets which satisfy the 
following conditions. If $m_{i}+1 \leq m \leq m_{i+1}$ then

(a) $Q_{m}$ has the form $Q_{m}=\left\{x \in Q: j_{k} 2^{-i}<x_{k}<\left(j_{k}+1\right) 2^{-i}, k=2, \ldots, n\right\}$ in $\mathrm{CS}_{1}$ for some integers $j_{2}, \ldots, j_{n}$,

(b) $0 \notin \partial Q_{m}$,

(c) $\sup _{x \in Q_{m}}(h(x)-v \cdot x)^{+}>2^{-i-3}$,

(d) the set $Q_{m}$ is disjoint from all sets $Q_{l}, l \leq m_{i}$.

Since the function $(h(x)-v \cdot x)^{+}$is Lipschitz with the constant $K_{1}>1$ we have $(h(x)-v \cdot x)^{+} \geq 2^{-i-4}$ for all $x$ in the set $V_{m}=\left\{z \in Q_{m}:|z-\hat{x}|<2^{-i-4} / K_{1}\right\}$ where $\hat{x}=\hat{x}(m)$ is a point in the closure of $Q_{m}$ where the supremum in (c) is attained.

Let $a_{m}=\inf _{x \in Q_{m}}|x|$ and $b_{m}=\sup _{x \in Q_{m}}|x|$ and note that $b_{m} \leq a_{m} 2(n-1)^{1 / 2}$ by (b). The volume of $V_{m}$ is at least $\left(2^{-i-5} / K_{1}\right)^{n-1}$ and therefore

$$
\begin{aligned}
\int_{Q_{m}} \frac{(h(x)-v \cdot x)^{+}}{|x|^{n}} d x & \geq \int_{V_{m}} \frac{(h(x)-v \cdot x)^{+}}{|x|^{n}} d x \\
& \geq 2^{-i-4}\left(2^{-i-5} / K_{1}\right)^{n-1} b_{m}^{-n} \\
& \geq 2^{-i-4}\left(2^{-i-5} / K_{1}\right)^{n-1} a_{m}^{-n} 2^{-n}(n-1)^{-n / 2} \\
& =\left(2^{-6 n+1} K_{1}^{-n+1}(n-1)^{-n / 2}\right)\left(2^{-i n} a_{m}^{-n}\right) .
\end{aligned}
$$

Let

$$
Q_{m}^{1}=\left\{x \in \mathbf{R}_{2}^{n-1}: \operatorname{dist}\left(\tilde{x}, Q_{m}\right) \leq 2^{-i-1}\right\}
$$

and

$$
Q_{m}^{2}=\left\{x \in \mathbf{R}_{2}^{n-1}: \operatorname{dist}\left(\tilde{x}, Q_{m}\right) \leq 2^{-i-2}\right\}
$$

where $\tilde{x}$ is the projection in $\mathrm{CS}_{1}$. The function

$$
f_{m}(x)=\left[2^{-i-2}-\operatorname{dist}\left(x, Q_{m}^{2}\right)\right]^{+}
$$

defined for $x \in \mathbf{R}_{2}^{n-1}$ is Lipschitz with constant 1. It follows from (2.9) and (c) that $\sup _{x \in Q_{m}}(h(x)-v \cdot x)^{+}<2^{-i-2}$ so

$$
\begin{gathered}
\left\{x \in \mathbf{R}^{n}: v \cdot \tilde{x}<x_{1}<h(\tilde{x}) \text { and } \tilde{x} \in Q_{m} \text { in } \mathrm{CS}_{1}\right\} \\
\subset\left\{x \in \mathbf{R}^{n}: 0<x_{1}<f_{m}(\tilde{x}) \text { in } \mathrm{CS}_{2}\right\} .
\end{gathered}
$$

The support of $f_{m}$ is contained in $Q_{m}^{1}$ which has a volume no greater than $\left(|v|^{2}+1\right)^{1 / 2}\left(2^{-i+1}\right)^{n-1}$. Thus

$$
\begin{aligned}
\int_{\mathbf{R}_{2}^{n-1}} \frac{f_{m}(x)}{|x|^{n}} d x & \leq 2^{-i-2}\left(|v|^{2}+1\right)^{1 / 2}\left(2^{-i+1}\right)^{n-1}\left(a_{m} / 2\right)^{-n} \\
& =2^{2 n-3}\left(|v|^{2}+1\right)^{1 / 2}\left(2^{-i n} a_{m}^{-n}\right) .
\end{aligned}
$$

This and (2.10) show that

$$
\int_{\mathbf{R}_{2}^{n-1}} \frac{f_{m}(x)}{|x|^{n}} d x \leq c \int_{Q_{m}} \frac{(h(x)-v \cdot x)^{+}}{|x|^{n}} d x
$$

where $c$ does not depend on $m$. Define $g_{1}=\sup _{k} f_{k}$. Since

$$
\left\{x \in Q:(h(x)-v \cdot x)^{+}>0\right\} \subset \bigcup_{k=1}^{\infty} \overline{Q_{k}}
$$


it follows from (2.11) that $D_{1}=\left\{x: x_{1}>g_{1}(\tilde{x})\right.$ in $\left.\mathrm{CS}_{2}\right\}$ satisfies the condition stated in the theorem. We have

$$
\begin{aligned}
\int_{\mathbf{R}_{2}^{n-1}} \frac{g_{1}(x)}{|x|^{n}} d x & \leq \sum_{k=1}^{\infty} \int_{\mathbf{R}_{2}^{n-1}} \frac{f_{k}(x)}{|x|^{n}} d x \leq c \sum_{Q_{k}} \int_{Q_{k}} \frac{(h(x)-v \cdot x)^{+}}{|x|^{n}} d x \\
& \leq c \int_{\mathbf{R}_{1}^{n-1} \cap\{|x| \leq 1\}} \frac{(h(x)-v \cdot x)^{+}}{|x|^{n}} d x<\infty . \quad \square
\end{aligned}
$$

ProOF OF THEOREM 2.2. (i) Suppose that (2.4) holds. Let the coordinate system $\mathrm{CS}_{2}$ in Lemma 2.1 be defined such that $v=\nabla h(0)$. It follows from (2.4) that (2.5) and (2.7) are satisfied. Define $D_{1}$ and $D_{3}$ as in (i) and (iii) of Lemma 2.1. Choose $\varepsilon>0$ so small that $D_{1} \cap U \subset D \cap U \subset D_{3} \cap U$ where $U=\left\{z:\left|z-x_{0}\right|<\varepsilon\right\}$. Define $D_{4}=D \backslash\left(\bar{U} \backslash D_{1}\right)$ and $D_{5}=D \cup\left(U \cap D_{3}\right)$. If $H_{*}^{x_{0}}$ is a standard excursion law in $D_{*}^{2}$ then by Lemma 2.1 and Theorem 2.1

$$
H_{4}^{x_{0}}=D_{*}^{2}-D_{4} \mathrm{KAC}-H_{*}^{x_{0}} \text { and } H_{5}^{x_{0}}=D_{*}^{2}-D_{5} \mathrm{KAC}-H_{*}^{x_{0}}
$$

are standard excursion laws. Let $H_{1}^{x_{0}}=D_{*}^{2}-D \mathrm{KAC}-H_{*}^{x_{0}}$. By definition, the excursion law $H_{1}^{x_{0}}$ is locally flat.

Fix a nonempty open ball $B \subset D_{4}$. We have $D_{4} \subset D \subset D_{5}$ and

$$
0<H_{4}^{x_{0}}\left(T_{B}<\infty\right) \leq H_{1}^{x_{0}}\left(T_{B}<\infty\right) \leq H_{5}^{x_{0}}\left(T_{B}<\infty\right)<\infty
$$

so $H_{1}^{x_{0}}$ is a standard excursion law in $D$ and therefore $H_{1}^{x_{0}}=H^{x_{0}}$. It follows that $H^{x_{0}}$ is locally flat.

(ii) Assume that $H^{x_{0}}$ is locally flat. Suppose that $H^{x_{0}}$ shares the local properties with the standard excursion law $H_{*}^{x_{0}}$ in $D_{*}^{2}$, where $D_{*}^{2}$ is the upper half-space in some coordinate system $\mathrm{CS}_{2}$. We can assume that $x_{0}=0$ in $\mathrm{CS}_{2}$ and that $D_{*}^{2}$ has the form $\left\{x: x_{1}>v \cdot \tilde{x}\right.$ in $\left.\mathrm{CS}_{1}\right\}$. We will prove that $\mathbf{R}_{2}^{n-1}$ is a tangent to $\partial D$ at $x_{0}$, that $\nabla h(0)$ exists and equals $v$, and (2.4) holds.

Suppose that (2.6) is satisfied and let $D_{2}$ be defined as in part (ii) of Lemma 2.1. It follows from part (i) of the proof of Theorem 2.1 and Lemma 2.1(ii) that

$$
H_{*}^{x_{0}}\left(T_{\partial D_{2}} \neq 0\right)=0 \text {. }
$$

For some neighborhood $U$ of $x_{0}, \partial D_{2} \cap U \cap D \cap D_{*}^{2}=\varnothing$ and $H^{x_{0}}$-excursions stay in $U \cap D \cap D_{*}^{2}$ for some interval of time $(0, T), T>0$ and therefore

$$
H^{x_{0}}\left(T_{\partial D_{2}}=0\right)=0 \text {. }
$$

This contradicts (2.12) since the excursion laws $H^{x_{0}}$ and $H_{*}^{x_{0}}$ share the local properties. Thus (2.6) cannot be satisfied and therefore (2.5) holds.

Suppose that (2.5) holds and the integral in (2.7) is infinite. Repeat part (iii) of the proof of Theorem 2.1 with the following changes:

(a) redefine $D_{2}$ as $D \cap D_{*}^{2}$,

(b) for $x \in D_{*}^{2}$ redefine $\hat{x}, r_{1}, r_{2}$ and $Q$ as follows:

$$
\begin{aligned}
\hat{x} & =\left\{v \cdot \tilde{x}-\left(x_{1}-v \cdot \tilde{x}\right) / 4, x_{2}, \ldots, x_{n}\right\} \text { in } \mathrm{CS}_{1}, \\
r_{1} & =\left(x_{1}-v \cdot \tilde{x}\right) \cdot \min (1 / 16,1 /(8 K)) \text { in } \mathrm{CS}_{1}, \\
r_{2} & =2 r_{1}, \\
Q & =\left\{x \in D: x_{1}=2 v \cdot \tilde{x}-h(\tilde{x})>v \cdot \tilde{x} \text { and }|x| \leq \varepsilon \text { in } \mathrm{CS}_{1}\right\},
\end{aligned}
$$

where $\varepsilon>0$ is a suitable constant. 
With these modifications part (iii) of the proof of Theorem 2.1 shows that $H^{x_{0}}$ and $H_{*}^{x_{0}}$ do not share the local properties. This contradiction means that the negation of (2.7) cannot hold. We conclude that (2.5) and (2.7) are satisfied.

As in the proof of Lemma 2.1, it follows from (2.5) and (2.7) that

$$
\lim _{|x| \rightarrow 0}|h(x)-v \cdot \tilde{x}| \cdot|x|^{-1}=0 .
$$

Thus $\nabla h(0)=v$ and (2.5) and (2.7) imply (2.4).

REMARK 2.2. Theorems 2.1 and 2.2 generalize Theorem 4.3 of Burdzy [1].

\section{Brownian excursions in smooth domains.}

LEMMA 3.1. If $D$ is a $C^{1, \alpha}$-domain for some $\alpha>0$ then the condition (2.4) is satisfied for every boundary point.

Proof. Let $D$ be a $C^{1, \alpha}$-domain for some $\alpha>0$ and $x \in \partial D$. Choose a coordinate system $\mathrm{CS}_{1}$ such that $x=0$ in $\mathrm{CS}_{1}$, the boundary of $D$ is represented by a function $h$ near 0 and $\nabla h(0)=0$. We have $|h(x)| \leq c|x|^{1+\alpha}$ for some $c>0$ and all $x$ in $\mathbf{R}^{n-1} \cap\{|x| \leq 1\}$, by the assumption that the domain is $C^{1, \alpha}$. Thus,

$$
\int_{\mathbf{R}_{1}^{n-1} \cap\{|x| \leq 1\}} \frac{|h(x)|}{|x|^{n}} d x \leq \int_{\mathbf{R}_{1}^{n-1} \cap\{|x| \leq 1\}} \frac{c|x|^{1+\alpha}}{|x|^{n}} d x<\infty
$$

and (2.4) is satisfied.

Let $D \subset \mathbf{R}^{n}, n \geq 2$ be a $C^{1, \alpha}$-domain for some $\alpha>0$ and let $L$ be a continuous additive functional such that its associated measure is the surface area measure on $\partial D$ (for the existence of such an $L$, see Revuz $[\mathbf{1 5}, \mathbf{1 6}]$ ).

For each point $x \in \partial D$ construct an excursion law $H^{x}$ in $D$ as follows. Choose a coordinate system $\mathrm{CS}_{1}=\mathrm{CS}_{1}(x)$ as in the proof of Lemma 3.1. Let $H_{*}^{x}$ be the standard excursion law in $D_{*}^{1}$ starting from $x$ and normalized so that $H_{*}^{x}\left(T_{B}<\infty\right)=1$, where $B=\left\{x: x_{1}=1\right.$ in $\left.\mathrm{CS}_{1}\right\}$. (This is possible because $0<$ $H_{*}^{x}\left(T_{B}<\infty\right)<\infty$ by Theorem 3.3(iii) of Burdzy [1].) Define $H^{x}=D_{*}^{1}-D \mathrm{KAC}$ $H_{*}^{x}$.

THEOREM 3.1. The pair $(d L, H)$ is an exit system in $D$. All excursion laws $H^{x}, x \in \partial D$ are standard and locally flat.

Proof. All excursion laws $H^{x}, x \in \partial D$ are standard and locally flat by Lemma 3.1 and Theorem 2.2. The pair $(d L, H)$ is an exit system in $D$ by Theorems 4.4 and 5.1 of Burdzy [1]. Note that the crucial assumption $(D)$ of Theorem 4.4 is satisfied due to Lemma 3.1 proved above.

REMARKS 3.1. (i) The above theorem generalizes Theorem 5.1 of Burdzy [1] where the same conclusion was reached under the assumption that $D$ is a $C^{2}$ domain. The theorem cannot be further strengthened to $C^{1}$-domains as shown by the next proposition.

(ii) The above theorem holds under the assumption that $D$ or $\mathbf{R}^{n} \backslash D$ is convex, with no additional assumptions about the regularity of $\partial D$. See Theorem 5.1 of Burdzy [1] for a proof of this statement. Alternatively, in this case one can show that (2.4) is satisfied almost everywhere on the boundary of $D$.

(iii) In view of Remark 1.1, we see that for each $z \in \mathbf{R}^{n}, P^{z}$-a.s. the excursions of Brownian motion in $C^{1, \alpha}$-domains share the local path properties with 
Brownian excursions from hyperplanes. See Theorems 3.1, 3.2 and 3.3 of Burdzy [1] (especially Theorem 3.3(vii) and (viii)) for some path properties of excursions from a hyperplane.

Proposition 3.1. There exists a $C^{1}$-domain $D$ in $\mathbf{R}^{2}$ such that (2.4) is satisfied for almost no $x \in \partial D$. There exists an exit system $(d L, H)$ in $D$ such that no excursion law $H^{x}$ is locally flat.

PROOF. We will prove first that for every integer $k>0$ there exists a nondecreasing differentiable function $g_{k}:[0,1] \rightarrow \mathbf{R}$ such that $0 \leq g_{k}^{\prime}(x) \leq 2^{-k}$ for all $x$ and the following condition holds for $x$ in a set of measure greater than $1-2^{-k}$ :

$$
\int_{0}^{1} \frac{\left|g_{k}(x)+g_{k}^{\prime}(x)(y-x)-g_{k}(y)\right|}{|y-x|^{2}} d y \geq k .
$$

We will define the function $g_{k}^{\prime}$. This and the condition $g(0)=0$ will uniquely define the function $g_{k}$. Let $a=2^{-2} \exp \left(-k 2^{2 k+4}\right), b=a \cdot 2^{-k}, g_{k}^{\prime}(1)=0$, and for each integer $m \geq 0$, let

$$
\begin{aligned}
g_{k}^{\prime}(x) & =0 & & \text { if } x=m(a+b) \text { or } x=m(a+b)+a, \\
& =2^{-k} & & \text { if } x=m(a+b)+a+b / 2 .
\end{aligned}
$$

Extend $g_{k}^{\prime}$ to the whole interval $[0,1]$ in such a way that it is continuous and linear on every interval where it has not been defined above.

Consider an $x \in\left[0, \frac{1}{2}\right]$ such that $g_{k}^{\prime}(x)=0$. Then for $y \in\left[x, x+\frac{1}{2}\right]$ we have

$$
\left|g_{k}(x)+g_{k}^{\prime}(x)(y-x)-g_{k}(y)\right|=\left|g_{k}(x)-g_{k}(y)\right|=\int_{x}^{y} g_{k}^{\prime}(z) d z .
$$

It is elementary to check that the last integral is greater than $2^{-2 k-3}(y-x)$ for all $y \geq x+2(a+b)$. Therefore

$$
\begin{gathered}
\int_{0}^{1} \frac{\left|g_{k}(x)+g_{k}^{\prime}(x)(y-x)-g_{k}(y)\right|}{|y-x|^{2}} d y \geq \int_{x+2(a+b)}^{x+1 / 2} \frac{2^{-2 k-3}(y-x)}{|y-x|^{2}} d y \\
\quad=2^{-2 k-3}\left(\ln \frac{1}{2}-\ln 2(a+b)\right) \geq 2^{-2 k-3}\left(\ln \frac{1}{2}-\ln 4 a\right) \\
\quad=2^{-2 k-3}\left(\ln \frac{1}{2}+k 2^{2 k+4}\right)=2^{-2 k-3} \ln \frac{1}{2}+2 k \geq k
\end{gathered}
$$

It can be proved analogously that (3.1) holds for all $x \in\left[\frac{1}{2}, 1\right]$ such that $g_{k}^{\prime}(x)=0$. It is now easy to see from the definition of $g_{k}^{\prime}$ that (3.1) is satisfied for $x$ in a set of measure greater than $1-2^{-k}$. The proof of existence of the functions $g_{k}$ with the desired properties is complete.

For $x \in[0,1]$, let $h(x)=\sum_{k=1}^{\infty} g_{k}(x)$ and $h^{\prime}(x)=\sum_{k=1}^{\infty} g_{k}^{\prime}(x)$. It is easy to see that the functions $h$ and $h^{\prime}$ are well defined and $h^{\prime}$ is the derivative of $h$. The function $h^{\prime}$ is continuous since the series $\sum_{k} g_{k}^{\prime}$ of continuous functions converges uniformly. Thus $h$ is a $C^{1}$-function.

Let $h_{m}=\sum_{k=1}^{m} g_{k}$. The derivative of $h_{m}$ is piecewise linear so for $x$ outside a finite set we have

$$
\left|h_{m}(x)+h_{m}^{\prime}(x)(y-x)-h_{m}(y)\right| \leq c_{m}(x) \cdot|y-x|^{2} \quad \text { for all } y \in[0,1] .
$$

If (3.2) holds then

$$
\int_{0}^{1} \frac{\left|h_{m}(x)+h_{m}^{\prime}(x)(y-x)-h_{m}(y)\right|}{|y-x|^{2}} d y \leq \int_{0}^{1} \frac{c_{m}(x)|y-x|^{2}}{|y-x|^{2}} d y=c_{m}(x) .
$$


For every integer $j>0$ we will find a set $A_{j} \subset[0,1]$ such that the measure of $A_{j}$ is greater than $1-2^{-j}$ and for all $x \in A_{j}$ we have

$$
\int_{0}^{1} \frac{\left|h(x)+h^{\prime}(x)(y-x)-h(y)\right|}{|y-x|^{2}} d y>j .
$$

Let $f_{m}=h-h_{m}$. The function $g_{k}^{\prime}$ is zero on a set of measure greater than $1-2^{-k}$. Therefore the function $f_{m}^{\prime}=\sum_{k=m+1}^{\infty} g_{k}^{\prime}$ is equal to zero on a set of measure greater than $1-\sum_{k=m+1}^{\infty} 2^{-k}=1-2^{-m}$. Let $A_{j}$ be the set of all $x$ 's such that $f_{j}^{\prime}(x)=0$ and (3.2) holds with $m=j$. The measure of $A_{j}$ is greater than $1-2^{-j}$ and we will now show that (3.3) holds for all $x \in A_{j}$.

Fix a point $x \in A_{j}$. Choose $k$ so large that $k-c_{j}(x)>j$. Note that $g_{k}^{\prime}(x)=0$ and (3.1) holds for this choice of $x$ and $k$. Since all functions $g_{m}$ are nondecreasing and $k>j$ we have

$$
\left|f_{j}(x)-f_{j}(y)\right| \geq\left|g_{k}(x)-g_{k}(y)\right|
$$

Thus

$$
\begin{aligned}
\int_{0}^{1} \frac{\left|h(x)+h^{\prime}(x)(y-x)-h(y)\right|}{|y-x|^{2}} d y \\
\quad=\int_{0}^{1} \frac{\left|h_{j}(x)+f_{j}(x)+\left(h_{j}^{\prime}(x)+f_{j}^{\prime}(x)\right)(y-x)-h_{j}(y)-f_{j}(y)\right|}{|y-x|^{2}} d y \\
\quad=\int_{0}^{1} \frac{\left|h_{j}(x)+f_{j}(x)+h_{j}^{\prime}(x)(y-x)-h_{j}(y)-f_{j}(y)\right|}{|y-x|^{2}} d y \\
\quad \geq \int_{0}^{1} \frac{\left|f_{j}(x)-f_{j}(y)\right|-\left|h_{j}(x)+h_{j}^{\prime}(x)(y-x)-h_{j}(y)\right|}{|y-x|^{2}} d y \\
\quad=\int_{0}^{1} \frac{\left|f_{j}(x)-f_{j}(y)\right|}{|y-x|^{2}} d y-\int_{0}^{1} \frac{\left|h_{j}(x)+h_{j}^{\prime}(x)(y-x)-h_{j}(y)\right|}{|y-x|^{2}} d y \\
\quad \geq \int_{0}^{1} \frac{\left|g_{k}(x)-g_{k}(y)\right|}{|y-x|^{2}} d y-c_{j}(x) \\
\quad=\int_{0}^{1} \frac{\left|g_{k}(x)+g_{k}^{\prime}(x)(y-x)-g_{k}(y)\right|}{|y-x|^{2}} d y-c_{j}(x) \\
\geq k-c_{j}(x)>j .
\end{aligned}
$$

Since $j$ is arbitrary it follows that the set of $x$ 's for which the integral in (3.3) is finite must have measure zero.

Extend the function $h$ to the whole real line by

$$
h(x)=h(x-[x])+[x] \cdot h(1)
$$

where $[x]$ denotes the integer part of $x$. Let $D=\left\{x \in \mathbf{R}^{2}: x_{2}>h\left(x_{1}\right)\right\}$. It is easy to see that $D$ is a $C^{1}$-domain. Since the integral in (3.3) is infinite for almost all $x \in[0,1]$ it follows that $(2.4)$ is satisfied almost nowhere on the boundary of $D$.

Let $(d L, H)$ be an exit system in $D$ and let $A$ be the set of all points $x \in \partial D$ such that $H^{x}$ is locally flat. It follows from the first part of the proof that the set $A$ has null harmonic measure so for each $z \in \mathbf{R}^{n}, P^{z}$-a.s. no excursion of Brownian motion has its endpoint in $A$. By time reversal, $P^{z}$-a.s. no excursion has its starting 
point in $A$. Define $H_{1}^{x} \equiv 0$ for $x \in A$ and $H_{1}^{x}=H^{x}$ otherwise. It is easy to see that $\left(d L, H_{1}\right)$ is an exit system in $D$ and no excursion law $H_{1}^{x}$ is locally flat.

REMARKS 3.2. (i) The above proposition implies by the exit system formula (see Remark 1.1) that for each $z \in \mathbf{R}^{2}, P^{z}$-a.s. no excursion of Brownian motion in the region constructed in the above proof shares all the local path properties with excursions from a line. The following example should clarify this remark.

If $e_{t}(\cdot)$ is a nonconstant excursion in the halfplane $D_{*} \subset \mathbf{R}^{2}$ (see $\S 1$ for the definitions) then $e_{t}(0) \in \mathbf{R}^{1}$ and the straight line $\mathbf{R}^{1}$ is not hit immediately by $\left\{e_{t}(s), s>0\right\}$.

If $e_{t}(\cdot)$ is a nonconstant excursion in the region defined in the last proof then $\left\{e_{t}(s), s>0\right\}$ immediately hits every straight line passing through $e_{t}(0)$. This holds, of course, for all excursions on $P^{z}$-almost all paths for all $z \in \mathbf{R}^{2}$. We omit the easy proof of this fact (see also Example 6.1 of Burdzy [2]).

(ii) Proposition 3.1 shows that quasi-locally flat excursion laws (terminology of Burdzy [2]) cannot be replaced by locally flat excursion laws in an exit system even if the region is quite smooth.

4. Applications to minimal thinness and boundary behavior of Green functions. The reader is referred to Doob [7] for definitions of Green function, Martin topology, minimal Martin boundary, minimal thinness, minimal fine topology and related concepts. We recall that the minimal Martin boundary and topology may be identified with the Euclidean boundary and topology in bounded Lipschitz domains (see Hunt and Wheeden [10]).

Suppose that a Lipschitz function $h$ represents a region $D$ near $0 \in \partial D$ in a coordinate system $\mathrm{CS}_{1}$. Suppose that a hyperplane $\left\{x \in \mathbf{R}^{n}: x_{1}=v \cdot \tilde{x}\right.$ in $\left.\mathrm{CS}_{1}\right\}$ is equal to $\mathbf{R}_{2}^{n-1}$ in a coordinate system $\mathrm{CS}_{2}$.

THEOREM 4.1. (i) Suppose that

$$
\int_{\mathbf{R}_{1}^{n-1} \cap\{|x| \leq 1\}} \frac{(h(x)-v \cdot x)^{-}}{|x|^{n}} d x<\infty .
$$

Then $D_{*}^{2} \backslash D$ is minimal thin in $D \cup D_{*}^{2}$ at 0 if and only if

$$
\int_{\mathbf{R}_{1}^{n-1} \cap\{|x| \leq 1\}} \frac{(h(x)-v \cdot x)^{+}}{|x|^{n}} d x<\infty .
$$

(ii) Suppose that (4.2) holds. Then $D \backslash D_{*}^{2}$ is minimal thin in $D$ at 0 if and only if (4.1) is satisfied.

ProOF. (i) Suppose (4.1) holds. Let $H_{*}^{0}$ be the standard excursion law in $D_{*}^{2}$ and let $D_{1}=D \cup D_{*}^{2}$. Define $H_{1}^{0}=D_{*}^{2}-D_{1} \mathrm{KAC}-H_{*}^{0}$. The excursion law $H_{1}^{0}$ is standard by Theorem 2.2. Let $D_{2}=D \cap D_{*}^{2}$ and define $H_{2}^{0}=D_{*}^{2}-D_{2} \mathrm{KAC}-H_{*}^{0}$. Theorem 2.2 implies that $H_{2}^{0}$ is standard if and only if (4.2) is satisfied. In other words (4.2) holds if and only if $D_{*}^{2} \backslash D_{2}$ is not regular for $H_{*}^{0}$. The excursion laws $H_{*}^{0}$ and $H_{1}^{0}$ share the local properties so (4.2) holds if and only if $D_{*}^{2} \backslash D$ is not regular for $H_{1}^{0}$. This is equivalent to $D_{*}^{2} \backslash D$ being minimal thin at 0 in $D_{1}$ by Lemma 3.1 of Burdzy [3].

(ii) Suppose that (4.1) and (4.2) hold. Then the standard excursion law $H^{0}$ in $D$ is locally flat by Theorem 2.2. This excursion law shares the local properties 
with $H_{*}^{0}$ and in particular $D \backslash D_{*}^{2}$ is not regular for $H^{0}$. Lemma 3.1 of Burdzy [3] implies that $D \backslash D_{*}^{2}$ is minimal thin in $D$ at 0 .

(iii) Suppose now that (4.2) holds but (4.1) is not satisfied. We will assume that $D \backslash D_{*}^{2}$ is not regular for $H^{0}$ and will show that this assumption leads to a contradiction. Indeed, under this assumption $H_{3}^{0} \stackrel{\mathrm{df}}{=} D-D_{2} \mathrm{KAC}-H^{0}$ is a standard excursion law in $D_{2}$. Therefore $H_{3}^{0}=H_{2}^{0}$ and so $H_{3}^{0}$ is locally flat. We have $H^{0}=$ $D_{2}-D$ KAC- $H_{3}^{0}$ and it follows that $H^{0}$ is locally flat. This contradicts Theorem 2.2. We have shown that $D \backslash D_{*}^{2}$ is regular for $H^{0}$ and so by Lemma 3.1 of Burdzy [3], $D \backslash D_{*}^{2}$ is not minimal thin in $D$ at 0 .

REMARK 4.1. The above theorem generalizes Theorem 3.2 of Burdzy $[3]$ and Corollaries 4.2 and 4.3 of Burdzy [4] (see also Remark 4.2(ii) of the last paper). Alternative criteria for minimal thinness in a half-space were given earlier by Essen and Jackson $[\mathbf{9}]$.

For every domain $\tilde{D}$, extend the Green function $G_{\tilde{D}}(\cdot, \cdot)$ to $\mathbf{R}^{n} \times \mathbf{R}^{n}$ by declaring that $G_{\tilde{D}}(x, y)=0$ for $(x, y) \notin \tilde{D} \times \tilde{D}$.

Lemma 4.1. Suppose that $D_{1}$ and $D_{2}$ are Lipschitz domains, $y \in D_{1} \subset D_{2}$ and $x \in \partial D_{1} \cap \partial D_{2}$. Then $D_{2} \backslash D_{1}$ is minimal thin in $D_{2}$ at $x$ if and only if

$$
\operatorname{mf}_{\substack{z \rightarrow x \\ z \in D_{1}}} \frac{G_{D_{1}}(z, y)}{G_{D_{2}}(z, y)}>0
$$

Proof. The limits

$$
\operatorname{mf} \lim _{\substack{z \rightarrow x \\ z \in D_{1}}} \frac{G_{D_{1}}(z, y)}{G_{D_{2}}(z, y)} \text { and } \quad \limsup _{\substack{z \rightarrow D_{1} \\ z \in D_{1}}} \frac{G_{D_{1}}(z, y)}{G_{D_{2}}(z, y)}
$$

exist and are equal by Theorem 1.XII.14 of Doob [7]. It follows from Theorem 11 of Naim [13] that the limits are greater than 0 if and only if $D_{2} \backslash D_{1}$ is minimal thin at $x$ in $D_{2}$.

LEMMA 4.2. Assume the hypotheses of Lemma 4.1 hold and let $f$ be a function on $D_{2}$. If $D_{2} \backslash D_{1}$ is minimal thin in $D_{2}$ at $x$ then

$$
\mathrm{mf}-\lim _{\substack{z \rightarrow x \\ z \in D_{1}}} f(z)=\mathrm{mf}-\lim _{\substack{z \rightarrow x \\ z \in D_{2}}} f(z)
$$

if at least one side is well defined.

Proof. Let $P_{x}^{y}$ denote the distribution of Brownian motion in $D_{2}$ starting from $y \in D_{1}$ and conditioned to converge to $x$ at the lifetime. By Theorem 3.III.3 of Doob [7], the last exit time from $\partial D_{1}$ is strictly less than the lifetime of the process $P_{x}^{y}$-a.s. and therefore $P_{x}^{y}\left(T_{\partial D_{1}}=\infty\right)>0$. Let $\tilde{P}_{x}^{y}$ be the distribution $P_{x}^{y}$ conditioned by $\left\{T_{\partial D_{1}}=\infty\right\}$. Then $\tilde{P}_{x}^{y}$ is the distribution of Brownian motion in $D_{1}$ conditioned to converge to $x$. It follows that

$$
P_{x}^{y}\left(\lim _{t \rightarrow R} f\left(X_{t}\right)>a\right)=\tilde{P}_{x}^{y}\left(\lim _{t \rightarrow R} f\left(X_{t}\right)>a\right)
$$

since the above probabilities may take values 0 or 1 only (see 2.X.11(c) of Doob $[7])$. The last formula implies the lemma by Theorem 3.III.3 of Doob [7]. 
Recall the notation of Theorem 4.1. Let $N$ be the unit normal vector to $\mathbf{R}_{2}^{n-1}$ that points into $D_{*}^{2}$. Fix a point $x \in D \cap D_{*}^{2}$. Let

$$
\frac{\partial}{\partial N} G_{D} \equiv \frac{\partial}{\partial N} G_{D}(x, 0)=\lim _{\varepsilon \rightarrow 0} \frac{G_{D}(x, 0+\varepsilon N)}{\varepsilon}
$$

if the limit exists.

THEOREM 4.2. Assume the following interior cone condition:

$$
\left\{x \in \mathbf{R}^{n}: x \cdot N>a|\tilde{x}| \text { in } \mathrm{CS}_{2}\right\} \cap U \subset D
$$

for some $a>0$ and neighborhood $U$ of 0 in $\mathbf{R}^{n}$.

(i) Suppose that (4.1) holds. Then $\partial G_{D} / \partial N$ exists and $\partial G_{D} / \partial N<\infty$ and (4.2) is a necessary and sufficient condition for $\partial G_{D} / \partial N>0$.

(ii) Suppose that (4.2) holds. Then $\partial G_{D} / \partial N$ exists and $\partial G_{D} / \partial N>0$ and (4.1) is a necessary and sufficient condition for $\partial G_{D} / \partial N<\infty$.

PROOF. We will use the notation from the proof of Theorem 4.1.

(i) Assume that (4.1) and (4.2) hold. Let $D_{1}=D \cup D_{*}^{2}$. The set $D_{1} \backslash D_{*}^{2}$ is minimal thin in $D_{1}$ at 0 by Theorem 4.1 (ii) (replace $D$ by $D_{1}$ there). It follows that

$$
0<\operatorname{mf}-\lim _{\substack{y \rightarrow 0 \\ y \in D_{1}}} \frac{G_{D_{*}^{2}}(x, y)}{G_{D_{1}}(x, y)} \leq 1
$$

by Lemmas 4.1 and 4.2 and the fact that $G_{D_{*}^{2}}(x, y) \leq G_{D_{1}}(x, y)$. The set $D_{*}^{2} \backslash D$ is minimal thin in $D_{1}$ at 0 by Theorem $4.1(\mathrm{i})$. Therefore Lemmas 4.1 and 4.2 imply that

$$
\operatorname{mf}-\lim _{\substack{y \rightarrow 0 \\ y \in D_{1}}} \frac{G_{D}(x, y)}{G_{D_{1}}(x, y)}>0 .
$$

This combined with (4.4) shows that

$$
\operatorname{mf}-\lim _{\substack{y \rightarrow 0 \\ y \in D_{1}}} \frac{G_{D}(x, y)}{G_{D_{*}^{2}}(x, y)}>0
$$

and by Lemma 4.2

$$
\operatorname{mf}_{\substack{y \rightarrow 0 \\ y \in D_{*}^{2}}} \frac{G_{D}(x, y)}{G_{D_{*}^{2}}(x, y)}>0
$$

This implies that

$$
\lim _{\varepsilon \rightarrow 0} \frac{G_{D}(x, 0+\varepsilon N)}{G_{D_{*}^{2}}(x, 0+\varepsilon N)}>0 .
$$

To see this, follow the proof of Theorem 1.XII.21 of Doob [7] and use assumption (4.3). It is elementary to check that

$$
\lim _{\varepsilon \rightarrow 0} \frac{G_{D_{*}^{2}}(x, 0+\varepsilon N)}{\varepsilon}>0
$$

and this combined with (4.8) shows that $\partial G_{D} / \partial N>0$. 
When (4.1) holds but (4.2) is not satisfied then the limits in (4.5), (4.6), (4.7) and (4.8) are equal to zero and $\partial G_{D} / \partial N=0$.

(ii) Assume that (4.2) holds but (4.1) is not satisfied. Let $D_{2}=D \cap D_{*}^{2}$. The set $D_{*}^{2} \backslash D_{2}$ is minimal thin in $D_{*}^{2}$ at 0 by Theorem $4.1(\mathrm{i})$. Lemma 4.1 implies that

$$
\operatorname{mf}-\lim _{\substack{y \rightarrow 0 \\ y \in D_{2}}} \frac{G_{D_{2}}(x, y)}{G_{D_{*}^{2}}(x, y)}>0 .
$$

The set $D \backslash D_{*}^{2}$ and hence $D \backslash D_{2}$ is not minimal thin in $D$ at 0 , by Theorem 4.1(ii). Thus Lemma 4.1 implies that

$$
\operatorname{mf}-\lim _{\substack{y \rightarrow 0 \\ y \in D_{2}}} \frac{G_{D_{2}}(x, y)}{G_{D}(x, y)}=0
$$

and this combined with (4.10) yields

$$
\text { mf- } \lim _{\substack{y \rightarrow 0 \\ y \in D_{2}}} \frac{G_{D_{*}^{2}}(x, y)}{G_{D}(x, y)}=0 .
$$

Since $D_{*}^{2} \backslash D_{2}$ is minimal thin in $D_{*}^{2}$ at 0 , it then follows from Lemma 4.2 that

$$
\operatorname{mf}-\lim _{\substack{y \rightarrow 0 \\ y \in D_{*}^{2}}} \frac{G_{D_{*}^{2}}(x, y)}{G_{D}(x, y)}=0 .
$$

Now we deduce as in part (i) of this proof that

$$
\lim _{\varepsilon \rightarrow 0} \frac{G_{D_{*}^{2}}(x, 0+\varepsilon N)}{G_{D}(x, 0+\varepsilon N)}=0 .
$$

When combined with (4.9) this gives $\partial G_{D} / \partial N=\infty$.

REMARK 4.2. The last theorem is a stronger version of Corollaries 4.1, 4.2 and 4.3 of Burdzy [4] and generalizes some results of Widman [20] and Rippon [17].

ACKNOWLEDGMENT. The authors would like to thank Richard Olshen for useful discussions and the referee for several helpful comments.

\section{REFERENCES}

1. K. Burdzy, Brownian excursions from hyperplanes and smooth surfaces, Trans. Amer. Math. Soc. 295 (1986), 35-57.

2. __ Local properties of 2-dimensional Brownian excursions, preprint.

3. __ Brownian excursions and minimal thinness. Part I, Ann. Probab. (to appear).

4. ___ Brownian excursions and minimal thinness. Part II, Applications to boundary behavior of the Green function, Seminar on Stochastic Processes 1985, E. Cinlar, K. L. Chung, R. K. Getoor (Eds.), Birkhäuser, Boston, Mass. (to appear).

5. __ Brownian excursions and minimal thinness. Part III. Applications to the angular derivative problem, Math. Z. (to appear).

6. B. E. J. Dahlberg, Estimates of harmonic measure, Arch. Rational Mech. Anal. 65 (1977), 275-288.

7. J. L. Doob, Classical potential theory and its probabilistic counterpart, Springer, New York, 1984.

8. A. Dvoretsky and P. Erdös, Some problems on random walk in space, Proc. Second Berkeley Sympos. on Math. Stat. and Probability, Univ. of California Press, Berkeley, 1951, pp. 353-367. 
9. M. Essen and L. Jackson, On the covering properties of certain exceptional sets in a half-space, Hiroshima Math. J. 10 (1980), 233-262.

10. R. A. Hunt and R. L. Wheeden, Positive harmonic functions on Lipschitz domains, Trans. Amer. Math. Soc. 147 (1970), 507-527.

11. K. Itô and H. P. McKean, Diffusion processes and their sample paths, Academic Press, New York, 1965.

12. B. Maisonneuve, Exit systems, Ann. Probab. 3 (1975), 399-411.

13. L. Naim, Sur le rôle de la frontière de R. S. Martin dans le théorie du potentiel, Ann. Inst. Fourier (Grenoble) 7 (1957), 183-285.

14. S. C. Port and C. J. Stone, Brownian motion and classical potential theory, Academic Press, New York, 1978.

15. D. Revuz, Mesures associees aux fonctionelles additives de Markov. I, Trans. Amer. Math. Soc. 148 (1970), 501-531.

16. __ Mesures associees aux fonctionelles additives de Markov. II, Z. Wahrsch. Verw. Gebiete 16 (1970), 336-344.

17. P. J. Rippon, A generalisation of Widman's theorem on comparison domains for har monic measures, Ark. Mat. 17 (1979), 39-50.

18. T. Shiga and S. Watanabe, Bessel diffusions as a one-parameter family of diffusion processes, Z. Wahrsch. Verw. Gebiete 27 (1973), 37-46.

19. M. Shimura, $A$ limit theorem for two-dimensional conditioned random walk, Nagoya Math. J. 95 (1984), 105-116.

20. K.-O. Widman, Inequalities for the Green function and boundary continuity of the gradient of solutions of elliptic differential equations, Math. Scand. 21 (1967), 17-37.

21. D. Williams, Markov processes and martingales, vol. 1, Wiley, New York, 1979.

Department of Mathematics, University of California, SAn Diego, La Jolla, CALIFORNIA 92093 (Current address of R. J. Williams)

Current address: (Krzysztof Burdzy): Institute of Mathematics, Polish Academy of Sciences, ul. Kopernika 18, 51-617 Wrocław, Poland 